\title{
CONSIDERACIONES SOBRE LA PERCEPCIÓN DESDE LA PERSPECTIVA ENACTIVA
}

\author{
Considerations on Perception from the Enactive Perspective \\ ANA LORENA DOMÍNGUEZ ROJAS \\ Universidad Católica de Pereira, Colombia \\ ana.dominguez@ucp.edu.co
}

\begin{abstract}
This article reviews the enactive approach to perception, which defends the role of objects, the subject and the environment in the configuration of the phenomenal character of perception, that is, the qualitative dimension of experience. Initially the case of hallucination and its implications in the understanding of the phenomenal character of perception is retaken. Then, two positions within analytic philosophy of perception, representationalism and disjunctivism, are critically explored. Finally, enactivism is presented as a more promising alternative.
\end{abstract}

Key Words: Perception • hallucination • disjuctivism • intentionalism • enactivism

\section{Introducción}

Comprender la naturaleza de la percepción implica considerar la dimensión experiencial en la que el agente perceptivo asume un papel esencial, en la medida que algo es perceptivamente experimentado desde un punto de vista de primera persona. Esa dimensión cualitativa de la experiencia es también denominada carácter fenoménico de la percepción. Por ejemplo, escribiendo este texto y a través de la percepción que empleo para ir digitando una a una las palabras, experimento el mundo de una forma cualitativamente distinta de quien ahora lo lee. Esto es, el carácter cualitativo de la experiencia tiene que ver con lo que siente para alguien tener una experiencia perceptiva determinada.

Sin embargo, comprender la percepción no solo incluye hablar de la dimensión privada de la experiencia, sino también del lugar que ocupa el mundo y los objetos con sus variadas propiedades. Es decir, percibir el maullido de mi gato no es lo mismo que imaginarlo, aunque en algunas ocasiones el sonido pueda llegar a ser confundido. Mientras que, en la percepción, mi gato ocupa un lugar en el espacio y puede incluso ser escuchado por otras personas, no ocurre así cuando lo imagino (Thomas 2014). Surgen entonces las siguientes preguntas: ¿cómo está constituido 
este carácter fenoménico de la percepción? y ¿cuál es el papel que juegan los objetos en la configuración de este carácter?

La pregunta sobre el carácter fenoménico de la percepción y el papel que juegan los objetos en su configuración es problematizada dentro de la filosofía de la percepción en el contexto del argumento de la alucinación. El argumento plantea en pocas palabras la posibilidad de un caso de alucinación en el que dicha experiencia se presenta como fenoménicamente indistinguible de la percepción. Esto es, que para el sujeto no existe una diferencia en términos del cómo se siente estar en un estado perceptivo y uno alucinatorio. De acuerdo con Smith (2002), aparece entonces el problema de la percepción que refiere a la dificultad de entender que, si bien, la percepción es caracterizada por darnos un acceso al mundo, es problemático conciliar esto con la posibilidad de una experiencia fenoménicamente indistinguible en donde no hay objetos físicos presentes.

Así, dentro de la tradición analítica de la filosofía podemos ubicar dos posiciones frente al argumento de la alucinación que ofrecen respuestas al problema de la percepción: el representacionalismo y el disyuntivismo. El primero, estudia la percepción como un tipo de experiencia con un contenido intencional, cuyo carácter fenoménico está dado por cómo las cosas le aparecen al sujeto de determinada manera. Mientras que el segundo, describe a la percepción como un tipo de experiencia en donde se establece una relación con los objetos físicos y en donde el carácter fenoménico lo constituyen, en parte, las características físicas de éstos. Al respecto de estas dos posiciones se encuentran numerosos esfuerzos dentro de la filosofía por establecer claridad sobre la experiencia perceptiva y su relación con otros estados como la alucinación (Macpherson y Platchais 2013). Por ejemplo, desde el disyuntivismo encontramos un grupo de trabajos como los Martin (2009), Fish (2009) o McDowell (2008) que abordan aspectos como la naturaleza de la experiencia perceptiva o la relación entre percepción y conocimiento. De otro lado, en las teorías representacionales tenemos los trabajos de Tye (2014), Nanay (2015) o Pautz (2007) que abordan, entre otros temas, el contenido intencional de las experiencias perceptivas y su relación con los objetos físicos.

No obstante, es importante notar que el debate acerca de estos temas se extiende más allá de los límites de la filosofía. En las últimas décadas los diálogos más abiertos entre ésta y otras disciplinas han permito avanzar y nutrir la discusión sobre diversos fenómenos y la percepción, sin lugar a dudas, es uno de ellos. En el presente documento me propongo explorar una alternativa diferente a las posiciones de la filosofía analítica para tratar de comprender no solo la configuración del carácter fenoménico de la experiencia perceptiva, sino también su distinción respecto a la alucinación.

En este contexto, el enactivismo puede considerarse como una alternativa a las teorías analíticas clásicas, pero más alineada con la vertiente del disyuntivismo, que ofrece otras posibilidades en el estudio de la percepción y la configuración del carác- 
ter fenoménico. La teoría enactivista perteneciente a la familia de teorías en ciencias cognitivas denominadas 4E (por sus siglas en inglés enacted, embodied, extended y embedded), defiende no solo el papel de los objetos en la percepción, sino que también incorpora elementos como: la agencia corporal, el papel dinámico del entorno, el carácter histórico y situado de la percepción, la intersubjetividad, entre otros.

Mi meta aquí es proporcionar algunos argumentos a favor de la aproximación enactivista de la percepción. Mostraré en qué medida el enactivismo, como parte de las ciencias cognitivas, ofrece no sólo novedosas reflexiones conceptuales sobre la percepción, sino que también alienta a considerar a la investigación empírica como una pieza valiosa en la comprensión de la mente humana y sus procesos (Newen; Bruin y Gallagher 2018). Por consiguiente, dos de las principales contribuciones del documento son, por un lado, presentar una aproximación enactiva del carácter fenoménico de la percepción, que permita establecer una visión más integral de dicha experiencia, es decir, una que reconoce aspectos que habían sido omitidos por las visiones previas (e.g., el cuerpo y los objetos). Y de otro lado, mostrar líneas presentes y futuras de investigación sobre la percepción, en la cuales un dialogo entre la filosofía y las ciencias cognitivas es útil.

El orden de presentación será el siguiente: primero ofreceré algunas consideraciones iniciales sobre el caso de la alucinación y sus implicaciones en la caracterización de la percepción y el rol de los objetos. En la segunda parte presentaré dos posiciones sobre la percepción dentro de la filosofía analítica, el representacionalismo y el disyuntivismo, así como sus posibles limitaciones. Y finalmente, argumentaré a favor de la teoría del enactivismo, mostrando en qué medida resulta ser más apropiada para comprender la diversidad y complejidad de la experiencia perceptiva.

\section{Consideraciones generales sobre el caso de la alucinación}

En la filosofía de la percepción, el caso de la alucinación aparece incorporado dentro del argumento de la alucinación inicialmente empleado en una discusión epistemológica (Austin 1962; Ayer 1979). En este contexto, el argumento fue utilizado tanto para argumentar a favor de los datos sensoriales (entidades intermedias de naturaleza mental a las que accedemos directamente en la percepción) como para negar el realismo directo de la percepción (teoría que defiende un contacto directo a través de los objetos). En este apartado voy a revisar algunos aspectos provenientes de dos argumentos, el de Robinson (1994) y el de Crane y French (2005) como punto de partida de la discusión sobre la percepción y su carácter fenoménico.

La versión de Robinson presenta la posibilidad de una experiencia alucinatoria causalmente producida por la activación de los mismos procesos neuronales que hay en una percepción. Esto justamente es lo que caracteriza a esta versión como un 
argumento causal de la alucinación:

(i) Es teóricamente posible activar ciertos procesos cerebrales involucrados en la percepción exitosa para causar una alucinación, la cual es exactamente igual a la percepción en su carácter subjetivo (énfasis mío).

De acuerdo con Robinson, el argumento de la alucinación se basa en una noción de alucinación filosófica, que puede ser entendida como una experiencia causalmente producida y fenoménicamente indistinguible de la percepción. La alucinación filosófica es entonces "el tipo de experiencia que debería ocurrir si el sistema perceptivo y cerebral fueran estimulados de la misma manera en la que son estimulados en la percepción genuina, pero directamente y no por objetos externos" (Robinson 2013, p.313).

Según el mismo autor, existen dos principios a partir de los cuales es posible caracterizar una alucinación filosófica: el principio causal de que las mismas causas próximas tendrán los mismos efectos próximos y el principio de la superveniencia local del carácter fenoménico. Así, mientras que el primer principio refiere a la posibilidad de alterar el orden causal para producir ciertas experiencias indistinguibles, el segundo explica el carácter dependiente del nivel experiencial sobre el nivel neuronal (Pereira 2011).

De esta manera, el primer principio refiere a la posibilidad de manipular o alterar el orden causal cerebral que ocurre durante la percepción, para producir "artificialmente" una experiencia en ausencia de objetos distales. El principio mismas causas, mismos efectos está apoyado en los siguientes argumentos:

(i) Es posible activar las etapas intermedias de una cadena causal que normalmente nos lleva a percibir de un modo no-estándar (por ejemplo, mediante la estimulación directa de la retina, el nervio óptico o la corteza visual).

(ii) Si las etapas intermedias de una cadena causal se activaran de este modo no-estándar, de ninguna manera se alterarían las etapas posteriores de la cadena causal.

(iii) Si las etapas posteriores de la cadena causal estándar y las etapas posteriores de la cadena causal no-estándar fueran las mismas, entonces tendríamos como resultado una experiencia de la misma clase o naturaleza (Pereira 2011, p.120).

Si concedemos que es posible (gracias a la activación de los mecanismos cerebrales responsables de la percepción) obtener un tipo de experiencia con los mismos efectos que la percepción, entonces es factible considerar que ambas experiencias poseen un aspecto en común. No obstante, se requiere de otro elemento adicional y es que la alucinación se experimente como siendo una percepción, aun cuando ésta no lo sea. 
Así, aparece el segundo principio que refiere a la superveniencia local del carácter fenoménico, según el cual "las percepciones y sus contrapartes, las alucinaciones, poseen el mismo carácter fenoménico, ya que supuestamente el carácter fenoménico siempre sobreviene localmente en las causas proximales de la experiencia" (Pereira 2011, p.120). Básicamente, lo que se defiende es que, al haber una identidad en las causas próximas de una alucinación y una percepción, existe así un carácter fenoménico idéntico de ambos estados.

Al respecto, señala Robinson (1994) que si fuera posible fijar el estado neuronal que hay cuando tenemos la experiencia visual de una taza de café y luego activar estas mismas zonas removiendo al objeto, la persona seguiría teniendo la experiencia fenoménica de la taza de café. El cómo se siente una experiencia (un objeto como siendo redondo y rojo) estaría acompañado por ciertos procesos cerebrales, que al activarse artificialmente podrían dar lugar a una experiencia cualitativamente similar (de un tomate como siendo redondo y rojo) aun en ausencia del objeto. En este sentido, se argumenta que dos experiencias tengan un mismo antecedente causal con el mismo carácter fenoménico es una razón para defender un factor o naturaleza común entre estas experiencias, así como la idea de que los objetos no juegan un papel decisivo en las mismas.

Una fuente empírica de apoyo al principio de superveniencia del carácter fenoménico son los estudios realizados por Penfield sobre los años '30 (Fish 2010). Al respecto Fish (2010) es bastante crítico de dichos estudios, en los que parcialmente se apoya la posibilidad de una alucinación filosófica. Penfield estimuló eléctricamente la corteza cerebral de decenas de personas y recopiló reportes de primera persona sobre la experiencia mientras los sujetos eran estimulados sin objetos. Estos estudios llevaron a Penfield a sugerir que la estimulación cerebral es suficiente para producir la percepción, dado que, al activarse determinada zona del cerebro, las personas eran capaces de tener experiencias perceptivas de olores y sonidos en ausencia de objetos. "Es precisamente esta supuesta continuidad fenoménica entre percepciones y alucinaciones inducidas la que es utilizada por los filósofos (como Robinson) con el objetivo de establecer un factor común ontológico" (Pereira 2011, p.125).

Estos hallazgos de Penfield apoyan el principio de la naturaleza común de las experiencias bajo el presupuesto de que la alucinación y la percepción son producidas mediante la activación del mismo mecanismo cerebral, dando lugar a experiencias cualitativamente similares (Robinson 1994). Tal como lo recupera Pereira (2011) "la actividad neuronal inducida por Penfield parece ser suficiente para generar una experiencia con carácter fenoménico en un universo muy restringido de sujetos que ... son sujetos corporizados, situados en un entorno y que han tenido experiencias perceptivas previamente" (p.125). Es decir, no resulta suficiente considerar la posibilidad de activación de ciertos mecanismos cerebrales recoja en su esencia la experiencia perceptiva que involucra desde el contexto situacional, al sujeto corporizado en un 
espacio tiempo y a los objetos como recursos del ambiente (Fish 2009).

Esta forma de entender el carácter fenoménico de la experiencia perceptiva es discutible a la luz de la riqueza de la interacción que el perceptor establece con el mundo en la percepción. Experimentamos al mundo, perceptivamente hablando, desde una corporalidad activa (Gibson, 1979), que parece no vislumbrarse en este tipo de visiones que están apelando estrictamente a los mecanismos cerebrales de la percepción (punto sobre el que volveré más adelante).

Hasta este momento la indistinguibilidad fenoménica parece más un recurso que opera como un presupuesto filosófico poco discutido, pero ampliamente utilizado. Aun así, suponiendo que es posible tener una experiencia fenoménicamente indistinguible de la percepción, ¿es esta una razón suficiente para justificar que la alucinación y la percepción son de la misma naturaleza? En esta línea, la versión del argumento propuesta por Crane y French (2005) pone énfasis en el compromiso con el factor de tipo común que conduce al rechazo del carácter relacional de la percepción. Veamos.

(i) Parece posible que un sujeto tenga una experiencia - una alucinación - que sea subjetivamente indistinguible de una percepción genuina pero donde no hay un objeto independiente de la mente que se perciba.

(ii) La percepción y la alucinación subjetivamente indistinguibles son experiencias esencialmente del mismo tipo (énfasis mío).

(iii) Por lo tanto, no es posible que la esencia de la percepción dependa de que los objetos sean experimentados, ya que fundamentalmente la percepción es el mismo tipo de experiencia que aquella que ocurre en ausencia de los objetos.

(iv) Por lo tanto, la concepción cotidiana de la percepción - la cual trata a la experiencia como dependiente de que el sujeto establezca una relación con objetos independientemente de la mente - no puede ser correcta (Crane y French 2005, p.6).

La versión del argumento de la alucinación de Crane y French (2005) presenta claramente el problema de la percepción que se plantaba al inicio del texto (Smith, 2002). Esto muestra, en general, que señalar que la concepción cotidiana de la percepción nos da un acceso al mundo directo e inmediato es cuestionable. En el argumento de la alucinación se plantea que la relación con los objetos en la percepción no es una relación constitutiva en la medida que el carácter fenoménico de la percepción no depende directamente de la presencia de los objetos.

En el marco de la filosofía de la percepción analítica suele concederse la indistinguibilidad fenoménica asociada a la posibilidad de la alucinación (justificada desde un presupuesto); no obstante, el tema de debate se centra en los compromisos que conlleva aceptar la indistinguibilidad. Un ejemplo de esto es el compromiso acerca de la naturaleza de la experiencia perceptiva. Considero, en este sentido, que una 
estrategia para argumentar en contra del factor de tipo común es analizar más cuidosamente cómo está configurado el carácter fenoménico de la experiencia perceptiva, de tal manera que logre evidenciar la importancia del carácter relacional de la experiencia.

\section{Sobre el carácter fenoménico de la experiencia perceptiva en la filosofía analítica}

\subsection{Postura representacionalista}

El teórico representacionalista estudia los estados mentales (e.g., las experiencias perceptivas) como estados que son acerca de algo (Macpherson 2014). Este tipo de estados se sienten de determinada forma, es decir, que son estados con un carácter fenoménico. Según Byrne (2001) "cuando uno ve un gato, la experiencia es «acerca» del gato: éste es el componente representacional o intencional de la experiencia" (p.199). Es decir, que el tipo de objeto en el caso de la alucinación es un objeto intencional, justo como la daga de Macbeth (Thompson 2008).

Desde una visión representacionalista, el carácter fenoménico podría ser comprendido así:

cómo es (la experiencia), cómo se siente esta es un asunto de las propiedades que mi experiencia representa. Así, el carácter fenoménico está afuera en el mundo. Es también es cierto, incluso si estoy alucinando: solo que en este caso las propiedades externas relevantes no están instanciadas. (Tye 2015, p.484) (énfasis agregado).

Ahora, para algunos representacionalistas las percepciones constituyen estados representacionales análogos al caso de las creencias (Thompson 2008). Las creencias son tipos de estados mentales que tienen un contenido intencional, es decir, que son acerca de algo en el mundo. Como las creencias, las experiencias perceptivas tienen un contenido intencional (e.g., la rojez de la flor). Desde esta visión, más cercana a un representacionalismo de contenido, se podría defender un 'principio de tipo común' entre la percepción y la alucinación, señalando que en ambas experiencias se comparte el mismo carácter fenoménico, dado que éste depende del contenido representacional que puede ser el mismo en ambos casos (Thompson 2008). ${ }^{1}$ Es decir, que para un sujeto puede parecer lo mismo, fenoménicamente hablando, el percibir un tomate rojo que alucinar un tomate rojo. Es posible, no obstante, que el contenido intencional no sea satisfecho porque el objeto no exista (caso de alucinación) o porque el objeto físico no corresponda con las características del objeto experimentado (caso de ilusión). 
Es decir, incluso en la percepción, lo importante sobre lo objetos son las propiedades que la experiencia representa, no los objetos en sí. Mientras que en la percepción "las propiedades atribuidas en el contenido son propiedades que tiene el objeto visto" (Tye 2009, p.82), éste no es el caso de la experiencia de alucinación, donde las condiciones de corrección no son satisfechas porque el objeto material relevante está ausente. De acuerdo con Nanay (2015), es precisamente el hecho de que los estados perceptivos puedan fallar al representar correctamente lo que evidencia ciertas ventajas de una aproximación representacionalista respecto de una relacional (como el disyuntivismo).

Si bien, el carácter representacional de la experiencia perceptiva es tomado, de cierta manera, como una ventaja del representacionalismo respecto del disyuntivismo, hay ciertos asuntos que permanecen abiertos al debate. Uno de ellos es el compromiso internista de la percepción que se asume desde una visión representacional. En este sentido, aunque al interior del representacionalismo en sus versiones más reciente (Tye 2015), se ha buscado aclarar el rol de los objetos en el caso de la experiencia perceptiva, resulta difícil pensar que el papel de los objetos se restrinja esencialmente a ser las condiciones de verificación de la experiencia (Pautz 2007).

Otro aspecto tiene que ver con la igualdad fenoménica (que el sujeto experimente a la alucinación como cualitativamente idéntica a la percepción) que se establece más como un presupuesto filosófico necesario y poco discutido, particularmente, a la luz de la diversidad y la complejidad de la experiencia perceptiva y alucinatoria (Macpherson y Batty 2016).

Finalmente, cabe resaltar que en la explicación representacionalista no es claro el papel que juega el cuerpo en la estructuración de la experiencia perceptiva, más allá de actuar como puente a través de los canales sensoriales. El llamado, como se hará en la tercera sección, es a comprender el efecto que tienen las experiencias corporales en la manera en cómo experimentamos el mundo en la percepción (Gallagher 2011).

\subsection{Postura disyuntivista}

Coincidentes con el llamado del realismo ingenuo, un conjunto de posiciones organizadas bajo el nombre de 'disyutivistas' realiza ciertas consideraciones sobre la percepción y el caso de la alucinación. Mientras que algunos disyuntivistas aceptan como posible el caso de una alucinación filosófica, consideran que la indistinguibilidad no es razón suficiente para justificar una naturaleza común, ya que la igualdad fenoménica puede ser explicada por otras razones (Soteriou 2009). ${ }^{2}$ De acuerdo con Vega-Encabo (2010):

una experiencia perceptiva de un campo de pasto verde es esencialmente verídica, si y solo sí, en la perspectiva $P$ bajo la cual el campo de pasto verde 
aparece fenoménicamente a $S$, la experiencia pone a $S$ en contacto con un campo de pasto verde (p.284).

Esto es, el carácter fenoménico de la experiencia perceptiva es distintivo y dependiente la presencia de los objetos. El disyuntivismo, de acuerdo con Vega-Encabo, se compromete con los siguientes principios sustantivos:

SP1. No hay un factor común entre la percepción y de alucinación.

SP2. Las experiencias perceptivas verídicas son esencialmente relaciones entre un perceptor, propiedades y objetos que son independientes del ámbito mental (Vega-Encabo 2010, p.286).

De acuerdo con Campbell (2009), el carácter fenoménico de la percepción, aparte de estar relacionado con los objetos, incluye también el punto de vista del sujeto, la modalidad de la experiencia perceptiva, la espacialidad y temporalidad, entre otros aspectos relacionales. En esta línea, Fish (2009) enfatiza que la diversidad y la complejidad en la organización del acto de la percepción son relevantes en la configuración del carácter fenoménico de la experiencia perceptiva. Desde su perspectiva, el carácter fenoménico está caracterizado por: a) la distribución y la organización de los objetos y las propiedades en el entorno, b) la posición que el sujeto ocupa desde un lugar del entorno, c) la naturaleza de la visión y d) la distribución de los recursos atencionales y conceptuales que posee el sujeto (Fish 2009, p.75). Tal como lo señala Martin (2009), la percepción no es únicamente la forma de obtener información acerca del mundo; en su carácter fenoménico incluye "literalmente" al mundo. Por estas y algunas otras razones y posiciones al interior del disyuntivismo, se distingue a la alucinación y a la percepción a la luz de su carácter fenoménico.

Entonces, ¿cuál es la estrategia del disyuntivista para explicar la indistinguibilidad fenoménica si rechaza el factor común?

Hay una intuición general de que nuestra capacidad introspectiva nos debería permitir captar la diferencia entre dos estados mentales si existe tal diferencia. Así que, si alguien es incapaz de distinguir entre la percepción y la alucinación, entonces es porque no hay tal diferencia después de todo. Esta reflexión es ampliamente cuestionada por Williamson (1990), quien afirma que un primer criterio para abordar la indistinguibilidad es la similitud. Esto es, que la indistinguibilidad entre dos estados o eventos se explica por la similitud que hay entre éstos. Asimismo, Williamson (1990) considera a la indistinguibilidad como un asunto de naturaleza epistémica, en razón de que bajo ciertos contextos de discriminación al sujeto no le es posible emplear el conocimiento necesario para poder distinguir entre dos eventos.

Para Williamson, la indiscriminabilidad consiste en lo siguiente: " $a$ es indiscriminable de $b$ para un sujeto en un tiempo, sí y solo sí, en ese tiempo el sujeto no es capaz de discriminar entre $a$ y $b$, esto es, sí y solo sí, en ese tiempo el sujeto no 
es capaz de activar (adquirir o emplear) el tipo relevante de conocimiento de que $a$ y $b$ son distintos" (Williamson 1990, p.8). De esta manera, la indiscriminabilidad es relativa a un sujeto y relativa a un tiempo: a) relativa al sujeto: porque un sujeto puede ser capaz de discriminar entre dos cosas que otro sujeto no puede discriminar, tal vez, porque el primero tiene mejor agudeza visual que el segundo, y b) relativa a un tiempo: porque un sujeto podría ser incapaz de distinguir entre dos cosas en un momento particular, pero podría distinguirlas en otro momento.

Mediante la objeción que realiza Williamson al supuesto de que el sujeto siempre tiene acceso a sus propios estados mentales, él señala que la capacidad de discriminar entre estados mentales ha sido sobrevalorada. A través de su argumento de la anti-luminosidad, Williamson demuestra que no todos los estados mentales pueden conocerse en primera persona, precisamente porque estos pueden tener cambios cualitativos imperceptibles para el sujeto. El contexto de discriminación se ve alterado por un amplio rango de factores (como lo relativo al sujeto o al tiempo) que inciden en que dos eventos o situaciones bastante diferentes no logren distinguirse.

En este sentido, de acuerdo con Fish (2009), no es necesario conceder el principio de tipo común a partir del supuesto de una experiencia alucinatoria con un carácter fenoménico que no se instancia en la experiencia. Ahora bien, Fish considera que la similitud en las creencias introspectivas de la percepción y la alucinación es el aspecto clave para comprender por qué alguien podría tomar un estado como la alucinación por otro como la percepción. La estrategia de Fish consiste en señalar que dado que la alucinación produce los mismos efectos cognitivos (tales como creencias, juicios, y comportamientos) que aquellos que habría producido una percepción genuina, entonces el sujeto no puede distinguirlas. Fish (2009) considera en esta medida que el principio de la superveniencia local está lejos de ser concluyente y actúa más bien como un supuesto del que dependen los defensores del principio de tipo común para apoyar la igualdad ontológica entre dos experiencias distintas (Fish 2008).

\section{Consideraciones enactivistas sobre la experiencia perceptiva}

En esta última sección haré énfasis sobre algunas ideas del enfoque enactivista sobre la percepción y especialmente sobre la configuración de su carácter fenoménico, ubicándome concretamente en el enactivismo sensorio-motor, a saber, un enfoque interesado en comprender a la percepción como una actividad realizada en una corporalidad en movimiento (Ward, Silverman y Villalobos 2017). El enactivismo es una aproximación dentro de las teorías 4E (Menary 2010), que enfatiza en el papel de los objetos, el carácter dinámico del entorno, el cuerpo y el movimiento (Froese y Di Paolo 2011; Varela 1990). Esta perspectiva busca comprender la relación de acople que 
se establece entre el objeto y el perceptor, las habilidades corporales/sensoriomotoras necesarias para un ajuste optimo en la interacción, entre otros elementos (Roberts 2010; O’Regan y Noë 2001). Esta visión de la percepción despierta el interés en aspectos de la experiencia perceptiva que habían sido omitidos de las visiones tradicionales en la filosofía de la percepción (Gallagher 2017) y que ahora toman fuerza gracias a una visión renovada, aunque minoritaria, de las ciencias cognitivas (Newen et al. 2018).

A través de los argumentos que presentaré a continuación mostraré en qué medida el enactivismo ofrece una visión holista de la experiencia perceptiva (Di Paolo, Buhrmann y Barandiaran 2017; O'Regan y Noë, 2001; Noë 2004) que nos conduce a encontrar razones para individuar la percepción de otras experiencias (e.g., la alucinación), al tiempo que nos permite enriquecer su caracterización. Estas razones, no son nuevas dentro del campo de la filosofía, pues muchas de ellas ya tenían sus orígenes en los trabajos de algunos fenomenólogos europeos. No obstante, sí resultan novedosas dentro del debate trazado por la filosofía analítica que he presentado en la segunda y tercera sección (Crane 2007).

\subsection{La percepción}

La percepción, vista desde la aproximación enactivista (como en la perspectiva ecológica de Gibson, por ejemplo), implica básicamente acción en el mundo. El cuerpo tiene, en este sentido, un rol activo y decisivo en la percepción a través del movimiento (Noë 2004). Por ejemplo, cuando un acróbata estima rápidamente una distancia para dar un salto en medio del aire. En este caso, la precisión en el movimiento del acróbata exige un tipo de habilidades adquiridas a través de la práctica corporal especializada (Avilés et al. 2014). No obstante, este nivel de maestría es del tipo que experimentamos en tareas más comunes como aprender a manejar carro, aprender a caminar o a preparar una receta. El cuerpo es el medio natural gracias al cual el sujeto manifiesta acciones intencionadas que generan acople óptimo con el entorno y concretamente con los objetos (Gallagher y Zahavi 2012).

A continuación, exploraré tres aspectos centrales para comprender la percepción dentro de la aproximación enactiva, a saber, los objetos, la corporeización del agente y el conocimiento perceptivo. Analizar estos elementos nos provee de buenas razones para pensar cómo el enactivismo representa ventajas en relación a las teorías tradicionales dentro de la filosofía de la percepción (Crane 2007). Las ventajas del enactivismo están orientadas esencialmente a entender a la percepción como una actividad cognitiva cuyo carácter corporizado configura el carácter fenoménico de la experiencia (Gallagher 2017; Degenaar 2013). 


\subsubsection{El rol de los objetos}

Retomando algunas consideraciones hechas por Dorsch (2010) y Farkas (2013), es factible establecer algunas características que posicionan claramente el papel de los objetos en la experiencia perceptiva.

La particularidad e independencia de los objetos: refiere al hecho de que los objetos se encuentran situados en un espacio independientemente de la experiencia que alguien pueda tener de ellos. La taza de café que ahora mismo es objeto de mi percepción es un objeto concreto; es esta taza y no otra la que está aquí presente ${ }^{3}$. Si bien, la fenomenología de la experiencia perceptiva puede cambiar dependiendo de la posición del sujeto respecto del objeto, este hecho no anula la existencia concreta del mismo.

De acuerdo con Noë (2005), una postura de la percepción debería ofrecer una explicación al dinamismo que se establece en la experiencia entre las constancias e inconstancias que se experimentan en la percepción respecto de los objetos. Cuando las cualidades cambian entonces la fenomenología de la experiencia también lo hace. Al respecto, Silverman (2018) considera que el cambio de las apariencias de los objetos, lejos de negar nuestro acceso directo a los objetos y sus cualidades, evidencia la importancia del acople activo que existe entre el sujeto corporizado y el objeto percibido en el acto perceptivo que ocurre en vivo.

Por su parte, la ubicación espacio-temporal de los objetos se refiere a que la experiencia perceptiva ocurre en el aquí y el ahora y es justamente esta constante actualización de nuestra interacción con el mundo lo que nos permite guiar nuestras acciones bajo cierta regularidad. Los objetos con los que interactuamos no están y en otro momento ya no, en este sentido se pueden trazar algunas diferencias entre el acto de percibir y el acto de imaginar un objeto (Ratcliffe 2013). Mientras que las imágenes mentales pueden mantenerse o permanecer en la conciencia por un esfuerzo o acto de atención y luego desvanecerse, nuestra experiencia nos indica que, al redirigir nuestra atención a otro punto del campo perceptivo, los objetos seguirán estando allí una vez se regrese a dicho punto (Thomas 2014).

Por otro lado, el carácter público de los objetos se refiere al hecho de que los objetos que ahora son parte de mi experiencia puedan también ser experimentados por otros sujetos. En esta línea, Beaton (2016) enfatiza el hecho de que, si bien el acceso a la experiencia en primera y tercera persona es diferente, en la percepción, nosotros tenemos un contacto y acceso a los objetos el cual no es fundamentalmente diferente de la experiencia que otros sujetos tienen sobre los mismos. En palabras de MerleauPonty (1975) "tengo, pues, conciencia de captar con el oído, y sobre todo con la vista, un sistema de fenómenos que no sólo constituyen un espectáculo privado, sino que es para mí e incluso para el otro el único posible, y ahí está lo que se llama real" (p.351). 
Adicional a esto, aparece la determinación de las propiedades sensoriales respecto de la constitución del carácter fenoménico de nuestra percepción. Cuando percibimos una fruta ésta se nos presenta con ciertas cualidades como, por ejemplo, la forma, el color, la textura, etc. Desde esta perspectiva, la explicación del carácter fenoménico de la percepción debe considerar a los objetos que están envueltos en la experiencia, así como también el punto de vista de la primera persona para quien la experiencia es significativa. En este sentido, existen acuerdos con la postura disyuntivista que asumen a los objetos como constitutivos del carácter fenoménico (Campbell 2009; Martin 2004).

$\mathrm{Al}$ respecto, Beaton (2016) señala que un enfoque enactivista considera que, si bien los objetos están constituidos por determinadas propiedades físicas, la riqueza de la experiencia perceptiva no se limita a éstas, sino que incluye también el papel que juega la estructura del sistema perceptivo del agente para capturar la riqueza del contacto con el mundo. En esta línea, el carácter fenoménico de la percepción debería ser considerado a la luz de la intersección entre cerebro-cuerpo-mundo, que describe más consistentemente la experiencia perceptiva de un agente corporizado (Gallagher 2011).

Finalmente, se presenta la direccionalidad, que es un aspecto decisivo para poder diferenciar la percepción de un sonido del recuerdo de un sonido, y refiere a la existencia u origen externo del objeto. En esta dirección, resulta problemático considerar que la simple activación cerebral constituya exhaustivamente la percepción, tal como lo cuestionaba el disyuntivismo frente al principio de superveniencia del carácter fenoménico. En palabras de Beaton (2013):

Cuando todo ocurre normalmente, y cuando nos damos cuenta que una mesa está justo ahí en frente de nosotros, sobre la base de nuestra experiencia, tenemos un conocimiento basado sobre la mesa en sí misma. Nuestra experiencia perceptual (y, por tanto, nuestro conocimiento) involucra constitutivamente a la mesa justo como esta aparece (p.305).

\subsubsection{Un sujeto corporizado}

Una consideración de la percepción desde el enfoque enactivista abraza en buena medida las tesis del realista directo enfocando su atención en cómo las habilidades sensoriomotoras ponen en contacto directo al sujeto y al objeto a través de la acción (Silverman 2018). Gracias a la percepción que posibilita una activa exploración de nuestro entorno somos capaces de generar un acople óptimo con el mundo. Este acople óptimo está dado, en parte, por el despliegue de determinadas habilidades sensoriomotoras coordinadas en función de las situaciones, de la posición del sujeto y de las características más relevantes de los objetos. De acuerdo con Roberts (2010) "Al estar en un episodio perceptivo dirigido por el mundo, el agente debe captar de 
alguna manera las consecuencias sensoriales de sus acciones" (p.102), esto es, cómo las maneras en que los inputs pueden estar organizados cambian, así como lo hace el mismo movimiento (Silverman 2018).

Así pues, la percepción no sólo depende de lo que acontece en el cerebro, sino que además involucra de manera importante la relación entre las diversas habilidades del perceptor y los objetos, siendo el cuerpo la vía natural-esencial que media en dicha interacción. La siguiente cita de Ratcliffe (2013) ilumina esta idea:

Cuando toco y soy tocado, partes de mi cuerpo pueden ser perceptivamente salientes en diferentes grados y de distintas maneras. Considere lo que sucede cuando usted está escribiendo y su mano empieza a cansarse. Se vuelve gradualmente más notorio e incómodo. A medida que esto sucede la mano se vuelve gradualmente menos efectiva como órgano de percepción y más como algo que se percibe (p.42).

Dicha idea conecta con el llamado del enfoque enactivista de considerar a la relación cuerpo-mundo como el punto de partida del conocimiento perceptivo, en la medida que entramos en contacto con los objetos por medio del uso de esquemas sensoriomotores.

Así que, la activación neuronal puede ser necesaria mas no suficiente para producir una percepción (Noë 2005), pues la riqueza y complejidad de la actividad cognitiva no se agota en la dimensión cerebral. Esto es, si percibir no es una actividad pasiva (Di Paolo 2016), sino que implica una interdependencia del sujeto con el mundo, los esquemas corporales de acción, íntimamente vinculados al funcionamiento de redes neuronales, constituyen una pieza importante (Silverman 2018).

Entonces, la constitución de nuestro sistema perceptivo juega un papel fundamental en nuestra percepción. En esta misma línea, tal como lo señala Fish (2010), para poner a prueba el principio de la superveniencia local sobre el carácter fenoménico, necesitaríamos primero un cerebro que estuviera totalmente libre de influencias externas para entonces determinar si la mera activación cerebral produce experiencias con carácter fenoménico. Y esto puede darse ciertamente en el plano de la experimentación mental mas no en el caso de la experiencia perceptiva, como de hecho tiene lugar.

\subsubsection{Conocimiento}

Desde una perspectiva enactivista, el conocimiento que se genera en la percepción no está asociado con conocimientos de naturaleza proposicional. De acuerdo con Beaton (2016), el conocimiento en la percepción está relacionado con un saber práctico del tipo ¿saber cómo?, que hace posible la interacción y el acople con el entorno y las características de los objetos. En palabras de Beaton (2016): 
Asi, por ejemplo, al percibir un objeto cercano yo conozco (implícitamente y no como un tipo de conocimiento explicito proposicional) cómo alcanzar y tocar las distintas partes este. E incluso para objetos más distantes sé cómo apuntar a las distintas partes de estos (p.266).

Es así como el conocimiento perceptivo sensoriomotor hace referencia a un saber de cómo actuar en determinadas situaciones a través de ciertas habilidades o destrezas corporales (Silverman 2018). Dicho saber, sin embargo, no implica un ejercicio consciente y deliberado sino más bien la capacidad en el momento de desplegar los esquemas de acción adecuados a la situación, dando sentido o haciendo significativa la experiencia (Avilés et al. 2014). Lo anterior, debido a que alcanzar un conocimiento habilidoso, como en el caso de un deportista, requiere de práctica para poder armonizar la intención con la ejecución de la acción. De acuerdo con Silverman (2018) el conocimiento sensoriomotor se constituye en una condición que hace posible el despliegue de la acción como por ejemplo agarrar una pelota o caminar con la ayuda de un bastón. A saber, es una "habilidad que fundamenta otras habilidades" (p.165).

La variación en las disposiciones sensoriomotoras es un ingrediente clave en la configuración del carácter fenoménico pues, aunque uno pueda tener dos experiencias con el mismo contenido (e.g., como se mencionaba al inicio del texto cuando se hablaba de percibir e imaginar un gato), el "cómo se siente" en términos de la acción será distinto en ambos casos al no existir un acople optimo a través del tacto o de la vista en el caso de la imaginación. Mas aún, al ser distintas las modalidades de interacción con los objetos (e.g., ver y tocar el gato) ello conduce necesariamente a una fenomenología diferente (Degenaar 2013). Es precisamente por esta razón, que la idea de que la activación cerebral por si sola es la responsable de la fenomenología de la experiencia perceptiva (Robinson 2013) es algo insostenible para el enactivismo (Di Paolo et al. 2017; Noë 2004). Siguiendo a Gibson (1979) las posibilidades de acción (affordances) que nos ofrece el entorno a través de las disposiciones de los objetos son aspectos decisivos en la comprensión de la constitución de la experiencia perceptiva. Esta visión ecológica de la percepción tiene en común con el enactivismo la restitución del papel significativo del ambiente y las interacciones del cuerpo con él.

Considerar la importancia del conocimiento a través de la acción abre líneas de trabajo muy interesantes para el enactivismo, por ejemplo, en lo que respecta a las interacciones primarias en el desarrollo humano. Estas interacciones vividas esencialmente a través del cuerpo están anudadas a los lazos afectivos originarios, especialmente, a aquellos creados con el cuidador (Di Paolo 2016; Zeedyk 2006). La riqueza del conocimiento perceptivo corporizado radica, en gran medida, en la existencia de otros con los cuales nos relacionamos y creamos lazos afectivos que nos configuran. La intersubjetividad, a saber, la dimensión de la experiencia que refiere a nuestro 
contacto con otras personas se realiza gracias a la existencia de una corporalidad afectiva vivida a través del movimiento (Gallagher 2013).

\subsection{Revisando los puntos centrales del problema de la percepción a la luz del enactivismo}

Los aspectos que previamente se han abordado, son de especial valor en la comprensión de la experiencia perceptiva y su distinción respecto de otro tipo de experiencia como la alucinación. Al inicio del texto señalaba que la alucinación filosófica refiere a un tipo de experiencia plausible, en donde al sujeto le parece estar percibiendo algo en ausencia de un objeto. El filósofo que emplea este tipo de experiencia, en general, no da cuenta de los mecanismos o procesos implicados en la producción de la alucinación, sino que simplemente asume su posibilidad (Martin 2004), a manera de recurso argumentativo. Así, tal como lo señala Thagard (2009), "si bien los experimentos mentales pueden ser herramientas útiles para desarrollar una hipótesis y contrastar esta con otras alternativas, ellos no son útiles para justificar la aceptación de la hipótesis" (p.250).

$\mathrm{Al}$ aproximarnos a la visión enactivista que explica cómo la fenomenología de la percepción depende, en buena parte, de nuestra capacidad de movimiento y conocimiento del mundo y los objetos, construido gracias a la interacción, resulta difícil entender que el carácter fenoménico sea explicado, esencialmente, por el contenido intencional de la experiencia, así como lo defiende el representacionalista. De acuerdo con Noë (2005), la caracterización de la percepción como un "hacer" (que involucra la participación del cuerpo y un conjunto de habilidades sensoriomotoras) choca con la idea de percepción internista (asociada a la posibilidad de la alucinación filosófica), que se vincula a aspectos representacionales o cerebrales. No obstante, el punto de la visión enactivista no está precisamente en negar ya sea los aspectos representacionales o cerebrales, sino más allá, poder argumentar a favor de una visión de la experiencia perceptiva que incluya lo corporal (que es más que lo cerebral) y que denote el rol esencial del entorno en donde podemos ubicar no solo objetos físicos, sino personas y sistemas sociales con los que interactuamos.

En este sentido, cabe señalar que los datos empíricos sobre la actividad neuronal no son, hasta el momento, lo suficientemente robustos para respaldar la tesis de la superveniencia local del carácter fenoménico y considerar que la fenomenología de la experiencia depende exclusivamente del cerebro (Ffytche 2013). Más allá, la investigación experimental ha permitido subrayar el importante papel que tiene la actividad cerebral, que de la mano del mundo y del movimiento, generarían una caracterización más holística de la experiencia perceptiva (Froese 2015; Gallagher 2011). Estas ideas son una vía promisoria de investigación que ayuda a comprender la interrelación entre cuerpo-cerebro-mundo en la explicación de la cognición 
humana (Gallagher 2017).

Ahora bien, el defensor del caso de la alucinación podría considerar que plantear la posibilidad de una alucinación filosófica no lo compromete a mostrar que dicha experiencia de hecho ocurre (Tye 2014). Basta con señalar un caso posible en donde al sujeto le parece estar percibiendo algo cuando no es así. El punto aquí es que en la experiencia perceptiva a un sujeto no le parece estar percibiendo a un tomate rojo, sino que efectivamente lo está percibiendo, a saber, está entrando en contacto con un objeto puesto en un entorno y lo hace a través de una acción corporizada en donde están implicadas tanto la propiedad del objeto como las disposiciones corporales del agente (Beaton 2016; Gallagher 2011; Silverman 2018).

Tal como lo señala Merleau-Ponty (1975), "el sujeto que alucina no ve, no oye en el sentido normal, utiliza sus campos sensoriales y su inserción natural en el mundo para fabricarse un medio ficticio con los escombros del mismo, conforme a la intencionalidad total de su ser" (p.354). Aunque un sujeto experimente el "ruido" de un tren acercándose cuando alucina, la experiencia de "escuchar" no se corresponde con un objeto en movimiento que cause un ruido y que se aproxima a él (Farkas 2013). Mientras que la alucinación parece conectar al sujeto con el mundo, en la percepción no solo parece, sino que de hecho ocurre (Beaton 2016).

\section{Conclusiones}

Los argumentos presentados en la segunda y tercera sección en dialogo con la apuesta enactivista de la cuarta sección nos permiten trazar varios puntos que no solo reflejan la importancia de las discusiones aquí presentadas, sino que invita a considerar líneas futuras de trabajo al respecto de estos temas. Por un lado, es posible señalar como una ventaja de la teoría enactivista sobre la percepción, la defensa que realiza del carácter relacional de la percepción. Es decir, el enactivismo no solo considera el papel de los objetos y sus propiedades físicas, como lo hace el disyuntivismo, sino que va más allá al tomar seriamente la corporeidad, el movimiento, la acción, el entorno y sus condiciones, en la configuración del carácter fenoménico de la percepción.

Por otro lado, también se recalca su interés en avanzar hacia una visión holista de la experiencia perceptiva que reconozca de forma enfática el entorno y que, por ende, se distancie de la visión internista plasmada en la visión representacionalista de la percepción. En este sentido, se rechaza la idea de que el carácter fenoménico de la percepción pueda ser explicado, estrictamente, por aspectos acaecidos al interior del cerebro o mecanismos representacionales. Finalmente, cabe mencionar que la teoría enactivista, por lo menos la variedad sensoriomotora revisada en este documento, reconoce a la percepción como anudada al movimiento y exploración corporal del mundo. En este sentido, la idea de plantear el caso de una experiencia alucinatoria 
con el mismo carácter fenoménico de la percepción, es más bien considerado un recurso filosófico argumentativo y no tanto así una vía para comprender cómo de hecho la experiencia ocurre.

Si bien, un propósito de la filosofía, particularmente de la filosofía analítica, es contribuir a la aclaración de conceptos que muchas veces resultan problemáticos, con el ánimo de generar argumentos e ideas mucho más precisas, este objetivo queda oscurecido por una tendencia a trabajar distanciados de los desarrollos en la ciencia. Tal como lo describe Thagard (2009), la clarificación conceptual que busca lograr la filosofía no puede convertirse únicamente en un ejercicio en un plano lingüístico, sino que debe reconocer los desarrollos en las ciencias con miras a generar explicaciones más robustas. En doble vía, la investigación empírica debería poder contribuir a dicha búsqueda de claridad conceptual.

\section{Referencias}

Austin, J. 1962. Sense and Sensibilia. Oxford: Oxford University Press.

Avilés, C.; Ruiz-Pérez, L.; Navia, J.; Rioja, N.; Sanz-Rivas, D. 2014. La pericia perceptivomotriz y la cognición en el deporte: del enfoque ecológico y dinámico a la enacción. Anales de psicología 30(2): 725-737.

Ayer, A. J. 1979. Los Problemas Centrales de la Filosofía. Trad. de R. Fernández. Madrid: Alianza.

Beaton, M. 2013. Phenomenology and embodied action. Constructivist Foundations 8(3): 298-313.

Beaton, M. 2016. Sensorimotor direct realism: how we enact our world. Constructivist Foundations 11(2): 265-276.

Byrne, A. 2001. Intentionalism defending. The Philosophical Review 110(2): 199-240.

Campbell, J. 2009. Consciousness and reference. In: B. P. McLaughlin, A. Beckermann, \& S. Walter (Eds.) The Oxford Handbook of Philosophy of Mind, pp.648-662. Oxford: Oxford University Press.

Crane, T. 2007. The problem of perception in analytic philosophy. Retrieved from https:// philpapers.org/rec/CRATPO-25

Crane, T; French, C. 2005. The problem of perception. The Stanford Encyclopedia of Philosophy. http://plato.stanford.edu/entries/perception-problem Substantive revision Fri Feb 4, 2011.

Degenaar, J. 2013. Perception from the phenomenal stance. Logique et Analyse 56(223): 273286.

Di Paolo, E. 2016. Participatory object perception. Journal of Consciousness Studies 23(5-6): 228-258.

Di Paolo, E., Buhrmann, T.; Barandiaran, X. E. 2017. Sensorimotor Life: An enactive proposal. Oxford: Oxford University Press.

Dorsch, F. 2010. The unity of hallucinations. Phenomenology and the Cognitive Sciences 9: 171-191. 
Farkas, K. 2013. A sense of reality. In: F. Macpherson \& D. Platchias (Eds.), Hallucinations: Philosophy and Psychology, pp.399-415. Cambridge: MIT Press.

Fish, W. 2008. Disjunctivism, indistinguishability, and the nature of hallucination. In: F. Macpherson \& A. Haddock (Eds.) Disjunctivism: Perception, Action, Knowledge, pp.144-167. Oxford: Oxford University Press.

Fish, W. 2009. Perception, hallucination, and illusion. New York: Oxford University Press.

Fish, W. 2010. Philosophy of perception. A contemporary introduction. New York: Routledge.

Ffytche, D. 2013. The hallucinating brain: neurobiological insight into the nature of hallucinations. In: F. Macpherson \& D. Platchias (Eds.) Hallucinations: philosophy and psychology, pp.45-63. Cambridge: MIT Press.

Froese, T. 2015. Enactive neuroscience, the direct perception hypothesis, and the social extended mind. Behavioral and Brain Sciences 38: 22-24.

Froese, T.; Di Paolo, E. 2011. The enactive approach, theoretical sketches from cell to society. Pragmatics and Cognition 19(1): 1-36.

Gallagher, S. 2011. Embodiment and phenomenal qualities: an enactive interpretation. Philosophical Topics 39(1): 1-14.

Gallagher, S. 2013. The socially extended mind. Cognitive Systems Research 25-26: 4-12.

Gallagher, S. 2017. Enactivist interventions: Rethinking the mind. Oxford: University Press.

Gallagher, S.; Zahavi, D. 2012. The phenomenological mind. Londres: Routledge.

Gibson, J. J. 1979. The ecological approach to visual perception. Boston: Houghto Mifflin.

Macpherson, F. 2014. Is the sense-data theory a representationalist theory? Ratio, https:// doi.org/10.1111/rati.12085

Macpherson, F; Platchais, D. 2013. Hallucination: Philosophy and psychology. Cambridge, MA: The MIT Press.

Macpherson, F.; Batty, C. 2016. Redefining illusion and hallucination in light of new cases. Philosophical Issues 26: 263-96.

Martin, M.G.F. 2004. The limits of self-awareness. Philosophical Studies 120(1- 3): 37-89.

Martin, M.G.F. 2009. The reality of appearances. In: H. Logue \& A. Byrne (Eds.) Disjunctivism: Contemporary Readings, pp.271-318. Cambridge: MIT Press.

Merleau-Ponty, M. 1975. Fenomenología de la percepción. México: Trillas.

Menary, R. 2010. Introduction to special issues on 4E cognition. Phenomenology and the Cognitive Sciences 9: 459-463.

Nanay, B. 2015. The representationalism versus relationism debate: explanatory contextualism about perception. European Journal of Philosophy 23(2): 321-336.

Newen, A.; Bruin, L.; Gallagher, S. 2018. Historical roots, key concepts and central issues. In: A. Newen, L. Bruin \& S. Gallagher (Eds.) The Oxford handbook of 4E. Oxford: Oxford University Press.

Noë, A. 2004. Action in Perception. Cambridge MA: MIT Press.

Noë, A. 2005. Real presence. Philosophical Topics 33: 235-264.

O’Regan, J. K.; Noë, A. 2001. A sensorimotor account of vision and visual consciousness. Behavioral and Brain Sciences 24(5), 883-917.

Pautz, A. 2007. Intentionalism and perceptual presence. Philosophical perspectives 21: 495541.

Pereira, F. 2011. Percepción, discriminabilidad introspectiva y el principio de factor común. Filosofía Unisinos 12(2): 114-135. 
Ratcliffe, M. 2013. Touch and the sense of reality. In: Z. Radman (Ed.) The Hand: An Organ of the Mind, pp.131-157. Cambridge MA: MIT Press.

Roberts, T. 2010. Understanding 'sensorimotor understanding'. Phenomenological Cognitive Science 9: 101-11.

Robinson, H. 1994. Perception. London-New York: Routledge.

Robinson, H. 2013. The failure of disjunctivism to deal with "Philosopher Hallucination". In: F. Macpherson \& D. Platchias (Eds.) Hallucinations: philosophy and psychology, pp.313330. Cambridge: MIT Press.

Silverman, D. 2018. Bodily skill and internal representation in sensoriomotor perception. Phenomenology and the Cognitive Sciences 17: 157-173.

Smith, A. D. 2002. The problem of perception. Cambridge, MA: Harvard University Press.

Soteriou, M. 2009. The Disjunctive Theory of Perception. In: E. N. Zalta (Ed.) The Stanford Encyclopedia of Philosophy. http://plato.stanford.edu/entries/perception-disjunctive/

Thagard, P. 2009. Why cognitive science needs philosophy and vice versa. Topics in Cognitive Science 1: 237-254.

Thompson, B. 2008. Representationalism and the Argument from Hallucination. Pacific Philosophical Quarterly 89(3): 384-412.

Thomas, N. 2014. The multidimensional spectrum of imagination: Images, Dreams, hallucinations, and active, Imaginative Perception. Humanities 3: 132-184.

Tye, M. 2009. Consciousness Revisited. Cambridge, Massachusetts: MIT Press.

Tye, M. 2014. What is the content of a hallucinatory experience? In: B. Brogaard (Ed.) Does Perception have Content? pp.291-310. Oxford: Oxford University Press.

Tye, M. 2015. Yes, phenomenal character really is out there in the world. Fish, W

Varela, F. 1990. Conocer. Barcelona: Editorial Gedisa.

Vega-Encabo, J. 2010. Hallucinations for disjunctivists. Phenomenology and the Cognitive Science 9: 281-293.

Ward, D.; Silverman, D.; Villalobos, M. 2017. Introduction: The varieties of enactivism. Topoi 36: 365-375.

Williamson, T. 1990. Identity and discrimination. Oxford: Basil Blackwell.

Zeedyk, S. 2006. From intersubjectivity to subjectivity: the transformative roles of emotional intimacy and imitation. Infant and Child Development 15: 321-344.

\section{Notas}

${ }^{1}$ El representacionalismo puede dividirse según el tipo de relación que se establezca entre el componente (o contenido) representacional y el carácter fenoménico de la experiencia (para una ampliación de este debate ver Tye 2009).

${ }^{2}$ En Martin (2004) se trata de una propiedad epistémica negativa, la cual consiste en que el sujeto que tiene la alucinación no podría distinguirla de la percepción verídica correspondiente, al menos no solo por introspección. De otro lado, Fish (2009) considera que la alucinación no posee un carácter fenoménico ya que el carácter es dado a partir del compromiso relacional que establece el sujeto con los objetos en una actividad contextual.

${ }^{3}$ Podría considerarse un caso en el que la taza de café ubicada frente a mí fuera intercambiada por otra taza perceptivamente semejante sin que yo me diera cuenta. En esa situación 
podría pensarse que no tengo acceso a la particularidad de los objetos. Sin embargo, este hecho epistémico de mi acceso a la particularidad de los objetos no cuestiona el que ahora mismo mi experiencia es acerca de esta taza y no de cualquier otra. Mi percepción es ahora sobre la taza de café nueva y éste sigue siendo un objeto concreto y particular.

\section{Agradecimentos}

I thank the Catholic University of Pereira for the support in the postulation of the article. I also thank Professor Fredy Eduardo Vasquez for the correction of style. 